\title{
Artifacts and Their Functions
}

A. W. Eaton

The Oxford Handbook of History and Material Culture

Edited by Ivan Gaskell and Sarah Anne Carter

Print Publication Date: Jun 2020 Subject: History, Social and Cultural History

Online Publication Date: May 2020 DOI: 10.1093/oxfordhb/9780199341764.013.26

\section{Abstract and Keywords}

How do artifacts get their functions? It is typically thought that an artifact's function depends on its maker's intentions. This chapter argues that this common understanding is fatally flawed. Nor can artifact function be understood in terms of current uses or capacities. Instead, it proposes that we understand artifact function on the etiological model that Ruth Millikan and others have proposed for the biological realm. This model offers a robustly normative conception of function, but it does so naturalistically by employing our best scientific theories, in particular natural selection. To help make this case, it proposes "living artifacts" (organisms designed for human purposes through artificial selection) as a bridge between the artifactual and the biological realms.

Keywords: artifact, function, intention, purpose, exaptation, evolution, technology, teleology, tableware

A particular problem arises with artifacts, meaning extended things shaped at least in part by human action. At the most general level, the problem is that artifacts are mute: they cannot by themselves tell us anything about where they came from, or about how or by whom they were used, or even whether they are intact or unfinished or damaged or mere parts of a larger whole. Muteness may not a problem if one has only a purely aesthetic interest in the formal and material dimension of an artifact; for instance, one can in most cases appreciate the shimmering texture of an artifact's surface without knowing anything about its history. But if one wants to learn about the past from artifacts, then their stubborn silence is a considerable obstacle.

The most fundamental step in overcoming this obstacle is arguably figuring out what an artifact is for. Armed with this bit of knowledge, we can determine whether a particular specimen is a part or a whole, complete or damaged, exemplary or inferior, a prototype or a flawed copy, and so on. But-and here is the more refined version of the problem animating this chapter-how does one figure out what an artifact is for, especially in advance of being able to answer these other questions (about parts and wholes and such) which could easily seem conceptually prior? 
Far be it for a philosopher to have any practical advice about this matter! My aim, rather, is to elucidate the relevant conceptual terrain and shed light on this notion of "for-ness"; that is, I hope to elucidate the very idea that an artifact is for some particular activity yet not for other activities. I carefully and methodically distinguish between various ways of understanding "for-ness" or, as I prefer to call it, function. I argue that an adequate account of function cannot reduce to an artifact's uses or capacities, nor to its maker's intentions, and argue that instead we ought to conceive of artifact function on a model that is quite similar to that used to explain the functions of living things. To help make the case for this way of thinking about artifact function, I suggest that we look to living artifacts that have been shaped by artificial selection to help us bridge the gap between functions of artifacts and the function of natural things (including living things).

\section{(0.36) What Is Artifact Function and Why Should We Care?}

Imagine that a huge explosion has killed all life on Earth and destroyed much of human material culture, and that buildings, roads, books, computers, and so forth have been incinerated or reduced to rubble. Very few artifacts remain. Now imagine that an archaeologist from Mars, who knows almost nothing about human civilization, has come to Earth to retrieve and analyze the few remains of this strange culture. As she travels the planet, she uncovers and collects a few samples. Of particular interest to her are two virtually physically identical objects that she found in locations quite remote from one another. Imagine that these objects are so similar as to be indistinguishable at even a very detailed level of analysis (Figure 2.1).

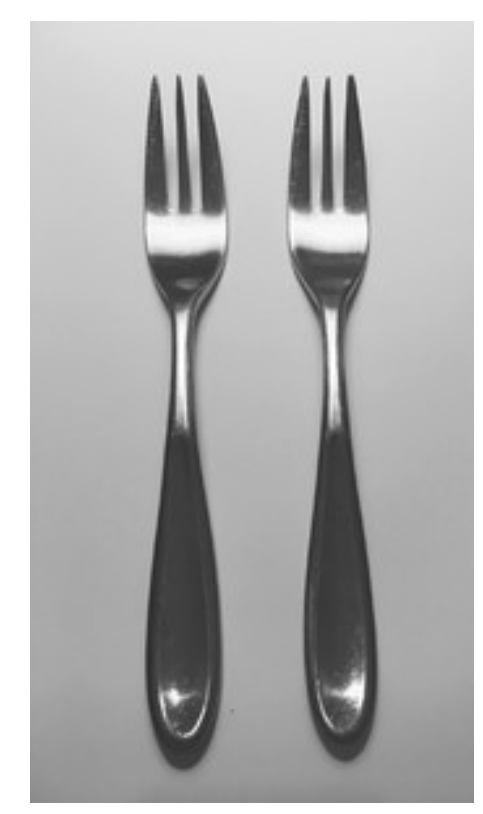

Figure 2.1. Two found objects. Photo credit: Cameron and Logan Eaton-Strong. 
The first thing our archaeologist wants to know is: Are these artifacts or mere freak accidents of nature? If artifacts, her next task is to classify them: What kind of thing are they? Once she's answered these questions, she will be able turn her attention to more complex (p. 37) questions regarding the structure of the concrete particular before her. Is it a single item-an individual unit in its entirety-or merely a part of one item, or one item fused to another? What are the artifact's component parts, and how do they relate to the whole they compose? Which of these parts are essential to its being the kind of thing it is and which are incidental? There are also normative questions to ask even at this basic fact-gathering stage: Is this particular item a good example of its kind? Or is it broken, malformed or otherwise defective, or worn down from years of use? Before our archaeologist can even begin to interpret these objects in their broader historical context, she must answer these very basic questions that are the province of what philosopher Daniel Dennett calls artifact hermeneutics, the enterprise of interpreting the human-made world. ${ }^{1}$

To begin to answer these basic questions, our archaeologist performs extensive postexcavation analysis of the objects' physical properties: she analyzes their formal attributes (e.g., dimension, shape, size, and looks for supervening aspects such as breakage patterns and cut marks) and surface attributes (e.g., color), identifies their chemical components, and dates them. We might expect that if all goes aright, such an analysis would yield everything required to answer our archaeologist's questions.

But this expectation is mistaken. An analysis of mere physical properties alone cannot answer even the basic typological question, namely, What is it? While the object's physical properties often yield negative constraints on typology-the objects pictured here could not be, for instance, trousers, tires, drinking vessels, or antifreeze-this tells us only what these objects are not; negative constraints reveal little about what they are. (Similar things could be said about dating these objects.) Although examination and analysis of an object's physical properties may be necessary for typology, no matter how powerful the microscope or extensive the chemical analysis, such examination will not by itself yield an answer to the most basic question, What is it? This is because, when it comes to artifacts, an object's identity, if you will, and its function are inextricably intertwined. Archaeologist Ian Hodder puts the point thus: "So how I define an entity depends on its use as a thing," 2 though by "use" Hodder means, or should mean, function. Hodder's point is evident in, for instance, our names for common artifacts-shoelace, nutcracker, hairbrush, windshield wiper, and so on, where the name of the artifact is the artifact's function. When dealing with artifacts, the best answer-that is, the answer with the most explanatory power-to the question "What is it?" points to the artifact's function.

Appeal to function is also how we pick out an artifact's component parts; indeed, function is what determines what counts as a part in the first place. ${ }^{3}$ The tires, seat, break cables, and chain are all are separable from each other and from the frame of my bicycle, yet they nevertheless count as proper parts of the bicycle; other connected-but-separable parts such as the basket and horn, however, do not. These determinations are based on 
our understanding of a bicycle's function: tires are essential to a bicycle's doing what it is supposed to do, whereas the basket and horn-useful as they may be-are not.

The central role of function in interpreting the human-made world probably strikes you as unexceptionable; after all, people do this all the time. However, there is a serious difficulty that any function-based account of artifact hermeneutics must overcome, namely to explain on what basis one properly ascribes a particular function to a given (p. 38) artifact. I emphasize "properly" here because when we ascribe a function to an artifact we can get it right or wrong. The central question here is, What makes an ascription of function correct or incorrect? On what basis do we say that someone has correctly described, or misdescribed, an artifact's function? These questions quickly lead us to a very basic question, namely: What, exactly, is a function and where is it located (both spatially and temporally) and what is our epistemic access to it? These questions, which are the focus of this chapter, are not merely of abstract philosophical interest; they must be answered, at least implicitly, before one can make claims about artifacts' functions in the first place. In this way, they should be of interest to anyone who hopes to use artifacts to learn about the past.

An examination of an object's physical properties alone, while perhaps necessary for ascribing a particular function to a given artifact, is insufficient for this purpose. Philosopher Elizabeth Anscombe argues that artifact functions, like intentions, are to be found in a wider context. ${ }^{4}$ However, since artifacts, unlike intentions, often endure diachronically and synchronically through a variety of contexts, the trick is to identify and demarcate the relevant context(s). Is every context in which an artifact finds itself relevant to its function? Or only certain contexts? If the latter, what makes some contexts especially relevant yet others irrelevant?

We begin in this section with criteria that an adequate account of artifact function should meet. First, such an account should be explanatory. It should tell us why the thing in question exists and why it has the shape and consistency that it does. As art historian Michael Baxandall puts it, Why at all and why thus? ${ }^{5}$ Second, it should be empirically adequate and historically accurate, reflecting current and past practices of making and using artifacts in particular contexts. Third and related, an adequate account of artifact function should allow for and be able to explain shifts in function over time (so that function is not conceived as an immutable or even stable feature of an artifact). Fourth, an account of artifact function should be broadly naturalistic in the sense that it should be consonant with and draw from our best science, including archaeology.

Fifth and finally, an account of artifact function should be normative. Since the concept of normativity has a bad reputation in some fields outside of philosophy, explanation is in order. I do not mean "normative" in a strong evaluative sense of the sort invoked in discussions of morality and aesthetics. As Ruth Millikan reminds us, "normative terms are not always evaluative, but can indicate any kind of measure from which actual departures are possible. ${ }^{\prime 6}$ It is this naturalistic and nonmoralizing sense of normativity that I mean to introduce as one criterion of an adequate conception of artifact function: that there is a 
performance of some activity (or activities) that is proper to the artifact in questionsomething that the artifact is supposed to do, even if it never performs this activity (perhaps because unable) or is occasionally co-opted for some other activity. ${ }^{7}$ This performance that is proper to the artifact provides a standard against which to evaluate the artifact's actual performances or its actual capacity to perform; it is what many philosophers refer to as a proper function. ${ }^{8}$ Such a standard-that is, a normative standard-is necessary in order for us to do the sorts of things that are common in the study of material culture, such as:

\section{(p. 39)}

- attribute a function to unused or rarely used artifacts,

- distinguish an artifact's accidental effects from its proper function,

- distinguish idiosyncratic and transient uses of an artifact from its function,

- account for malfunction and distinguish it from simply failing to have a function at all, and

- assess and explain defect and breakage in artifacts (essential to fixing or improving artifacts).

A normative account of artifact function conceives of functions in terms of performances that an artifact is supposed to achieve, even if it never achieves them. It means conceiving of functions in terms of purposes or, if you prefer, ends-that is, performance(s) for the sake of which the artifact exists, even though it may never realize, and may be chronically unable to realize, this performance(s).

It may strike you that the normativity requirement undercuts the naturalism requirement. After all, conceiving of artifact function in terms of purposes can easily appear vitalistic and anthropomorphizing, particularly given that our paradigmatic case of purposiveness is intentional action. Since artifacts are not intentional agents, attributing purposes to them could seem to posit a mind where there is none. Further, a normative understanding of function could seem to invoke backward causation (which is decidedly not naturalistic) in the sense that future outcomes-that is, the realization of some future performanceappear to explain present traits. Finally, this understanding of artifact function might seem empirically untestable precisely for the previous two reasons.

These are the kinds of worries that lead some, like the architect and industrial designer David Pye, to deny that functions of the sort proposed here (i.e., proper functions) really exist for artifacts. Pye writes: "Surely if there were activities proper to things, and if things acted, and if they had purposes, Newton might have been relied on to take note of these facts? 'Function' will not square with physics."9

In the third section, I offer an account of artifact function that answers these worries by reconciling normative demands with naturalistic ones. But before we can get there, we 
need to understand the problems with competing accounts of artifact function. These we explore in the next section.

\section{Two Theories of Artifact Function: Intentional- ism and Conventionalism}

What does our Martian archaeologist need to know in order (a) to determine whether her specimens have functions at all and, if so, (b) to ascribe functions properly? Before turning to what I think is the right answer in the third section, we first consider two popular but, I argue, misguided ways of construing artifact function: the first bases artifact function on the intentions of makers, and the second on the uses to which artifacts are put.

(p. 40) Philosophers who talk about function tend to focus exclusively on biological function. Where artifact function is discussed at all in this literature, intentionalism is the dominant view. Intentionalism holds that an artifact's function is determined by its maker's intentions; in particular, on the purpose(s) the maker intended the artifact to serve (and when the production process is divided between designers and fabricators, by "maker" one typically means the designer of the artifact). The attendant epistemological thesis holds that where an artifact's function is ambiguous, discerning the maker's intentions is the only surefire way to uncover the artifact's function. Valerie Hardcastle sums up the view: "The function of human artifacts depends on human intentions. We explicitly designed the microwave oven to heat food; all we have to do is talk to the microwave engineers to know what the microwave oven's function is."10

Intentionalism is a common-sense view that is motivated by what we might consider to be the typical process of making artifacts. The creation of an artifact often begins with a person's felt need or desire to achieve some end that, she hopes, can be fulfilled by means of some thing. With this end in mind (likely along with considerations of economy and aesthetics), she develops a plan according to which she modifies materials until she reaches the desired result, namely an object that fulfills her ends.

While it is true that the design of artifacts often centrally involves rational planning and intentional activity leading toward a goal, and also true that artifacts often function according to the goals that their makers set for them, this should not seduce us into accepting intentionalism. Shaping materials in a way that reflects the maker's goals may be an important part of the causal process that brings many, even most, artifacts into existence, but it would take magic or something like it for those goals to be transferred to, impressed upon, or deposited within an inanimate object. Understood as an ontological thesis, intentionalism is anti-naturalist.

Nor are artifacts always the result of rational planning in the way just described. Accident -in the sense of unintended and unforeseen occurrences-plays a decisive role in the designing of many common artifacts. In some cases, an accident occurs during the attempt to develop a kind of artifact that furthers the development of that very kind of artifact; for 
example, in the process of trying to develop vulcanized rubber, Charles Goodyear accidentally spilled a mixture onto a hot stove that resulted in the very thing he was hoping to produce. In other cases, an accident occurs in the attempt to develop one kind of artifact that results in the production of an entirely different artifact (that is, one with an entirely different function); for example, Teflon was the fortuitous result of a botched attempt to produce a new kind of refrigerant, and synthetic dye was the result of a failed attempt to produce a new treatment for malaria. In still other cases, an accident occurs and someone notices its potential; popsicles, for instance, were "discovered" when Frank Epperson accidentally left a container with a stick in fruit-flavored drink out all night in subfreezing temperature. In all such cases, an unintended and unforeseen incident plays a pivotal causal role in producing a trait that performs in a way deemed desirable, and this trait is then identified and selected to be reproduced in future artifacts on the basis of this desirable performance. I argue in the third section that this is (p. 41) a key to understanding how all artifacts acquire proper functions, regardless of accident's role (or lack thereof) in their production.

I have just said that, understood as an ontological thesis, intentionalism is anti-naturalist, but what of its attendant epistemological thesis? One could accept that intentions are not, strictly speaking, embedded in inanimate objects but still maintain that makers are often reliable guides to the functions of the artifacts that they produce. This is because, on my view, makers typically do the selecting and so they know which of an artifact's potential performances or uses serve as the basis for reproduction of the type (more on this in the third section).

But we should also note that makers are not infallible guides to the functions of artifacts they themselves have made. As I discuss in the following section, functions can and often do change over time, where artifacts acquire new functions that their makers had not anticipated. Consider, for instance, the so-called cigarette lighter receptacle in automobiles, which has come to serve primarily as an electric outlet for portable accessories (and only secondarily for cigarette lighters which are now typically offered as an optional extra-cost accessory). Sometimes artifacts' first functions are not even anticipated or endorsed by their makers; for example, Edison saw the phonograph (1877) as a tool for dictation, making "talking books" for the blind, teaching public speaking, preserving family sayings and the last words of the dying, creating new sounds for music boxes, making clocks that announce the time, recording telephone calls, and, almost as an afterthought, reproducing music (although he resisted the last of these as he entered the phonograph business a decade later). Finally, in the case of hermeneutically complex artifacts-like artworksthe maker may not even be the best interpreter of the artifact; in some cases, she may not even understand it. Indeed, artists are notoriously poor interpreters of their own work. The fact that the maker's intention is often a reliable indicator to an artifact's functionas with Hardcastle's microwave example-does not mean that intentions are constitutive of function. Like speaker meaning and utterance meaning, intended function and actual function can come apart. 
Turning to conventionalism, it should be noted that many anti-intentionalists favor a Wittgensteinian construal of function, namely that an artifact's function is simply its actual performances or the uses to which it is put. While this construal of function avoids the problems with intentionalism mentioned earlier, it cannot meet three of the criteria for an adequate conception of artifact function laid out in the previous section. Let me briefly remind you of these criteria and explain them in a bit more detail.

First, we want to retain a meaningful distinction between genuine function and merely using as. The fact that I use a particular fork exclusively as an implement to scrape burned food from pots and pans does not suffice to make pot scrubbing the proper function of my fork; the object remains a utensil for conveying solid food to the mouth, although it is one that I sometimes, or perhaps always, use as a pot scrubber.

Second, we want our understanding of function to be able to distinguish between function and merely accidental effects. A steel belt buckle, for instance, might serve to save a person from a bullet, but this propitious performance would be fortuitous and not (p. 42) the buckle's function. ${ }^{11}$ If our archaeologist were in possession of such an object and knew only that it had once saved a person from a bullet, she would be profoundly mistaken to interpret the object as a bullet stopper rather than a belt buckle.

Third, we want our formulation of function to be able to acknowledge cases of malfunction-in particular cases of defect and damage-and to be able to distinguish these cases from the case of simply not having a function at all. A broken artifact has been damaged and so can no longer perform its function (or perform it as well as it should), although it still may be able to do lots of other things; for example, a spoon with a hole in it will make a lousy spoon, but this will not diminish its use as a pot scrubber. But to say that it is a broken spoon is to say that it cannot do something that it should be able to do; that is, it has a function that it can no longer perform.

Can a conventionalist construal of function meet these demands? To start with, the conventionalist view should be formulated so as to preclude isolated or idiosyncratic uses. So the conventionalist might say that an artifact has the function of a particular kind of performance just in case the artifact regularly actually performs in that way. There will be some trouble in specifying what one means by "regularly," but let us imagine that this can be handled. The real problem for this formulation is that many artifacts are regularly used in ways that are not their functions. For instance, one survey reveals that three of every ten paper clips are lost, and that only one of every ten is actually used for holding papers together. ${ }^{12}$ Even though they are rarely used in this capacity, it is still the proper function of the kind of thing that we call "paper clips" to hold sheets of paper together.

Further, if saying that "this paper clip has the function of this holding sheets of paper together" means merely that "this paper clip actually regularly holds sheets of paper together," then this leaves no room for the concept of malfunction since, on such an account, lack of capacity to hold sheets together would be to have no function at all. Some are willing to bite this bullet: philosopher Christopher Boorse, for instance, insists that a broken artifact has no function until it is repaired. ${ }^{13}$ But what can "repair" mean, on this

Page 8 of 21

PRINTED FROM OXFORD HANDBOOKS ONLINE (www.oxfordhandbooks.com). (c) Oxford University Press, 2018. All Rights Reserved. Under the terms of the licence agreement, an individual user may print out a PDF of a single chapter of a title in Oxford Handbooks Online for personal use (for details see Privacy Policy and Legal Notice). 
account, if not restoring the artifact to a state in which it can function properly? This understanding of "repair" requires attributing to the artifact a function despite the fact that the artifact currently cannot-and in the case of deformation perhaps never could-perform in the requisite way. Pointing to the regularity of past performances or uses still does not help to deal with artifacts that were broken before they were ever used-say, for instance, something broken in the factory-nor with cases of artifacts that are deformed from the start.

To complicate matters further, many artifacts are regularly unused for a variety of reasons. Warehouses are full of artifacts whose uses expire before they ever make it out. There is furniture in the decorative arts sections of most museums that has never been used as furniture. The fire extinguisher in my kitchen has never, thank goodness, put out a fire, nor has the bumper on my automobile ever absorbed impact in a collision. About such examples you might be tempted to say that although they never get used in their lifetimes, they all have a capacity to perform in particular ways that constitute their functions. One doesn't have to be sitting in a chair most of the time, or ever, for the chair to have the capacity to support humans in the sitting position. But as already (p. 43) noted, most artifacts offer a wide spectrum of capacities that are not their functions: the fork has the capacity to be a pot scrubber just as the clothespin has the capacity to serve as an excellent bag closure. And once again, the malfunction requirement poses problems since broken and deformed artifacts lack the requisite capacities.

Perhaps we can tighten up the notion of "capacity" to mean "potential to be used in a certain way under normal conditions" and reformulate our conventionalist construal of artifact function accordingly: an artifact has the function of a particular kind of performance just in case the artifact has the capacity to perform in that way under normal conditions. The difficulty here is how to interpret "normal conditions." "Normal" cannot be understood in a statistical sense since, as we saw in the case of paper clips, a large majority of uses may be other than the artifact's proper function. Nor can we use a purely statistical construal of "normal" in the case of typically unused artifacts like car bumpers and fire extinguishers, since their functions are realized only under conditions that are extraordinary. Further, some artifacts-like shotgun pellets, most of which fly off into the air without hitting anything ${ }^{14}$-regularly fail to perform their function.

To construe "normal" as something like "under appropriate conditions" does not help, for what we want to know is how to distinguish the many adventitious uses of an artifact from those that constitute the artifact's function. To say that the artifact's function is just those uses that are appropriate begs the question. ("To beg the question" in philosophical parlance is to assume the very conclusion that one is trying to prove. In this case, it is precisely the question "Which uses count as appropriate?" that we are trying to explain here.)

It is this kind of worry that pushed philosopher Robert Cummins to insist that one must specify a system to which the artifact belongs. ${ }^{15}$ Mutatis mutandis, Cummins's view can be formulated this way: An artifact has the function of a particular kind of performance 
just in case the artifact actually performs in that way in the system of which it is a part. Let us for the moment set aside the thorny question of what makes an artifact part of one system rather than another. Even assuming this, merely being part of a system does not suffice to distinguish between proper function and adventitious effects. Consider this example from Larry Wright: imagine that a small nut in an engine comes loose and falls into just the right position under the valve-adjustment screw so as to properly adjust the poorly adjusted valve. Despite the fact that an internal combustion engine is a paradigmatic example of a complex system, and that the loosened nut is part of that system and affords proper valve adjustment that is necessary for the system's correct operation, we nevertheless should not say that valve adjustment is the proper function of the loosened nut. ${ }^{16}$

One might respond that these worries confuse tokens (particular instances of a thing) with types (general classes of things). While token artifacts may get used in all sorts of idiosyncratic ways, or not used at all, artifacts do not get their functions from individual uses; rather, so this line of thought goes, token artifacts get their function from their membership in a more general category of artifact type. So although the token clothespins in my house are used as bag closures, these idiosyncratic uses do not alter the clothespins' proper function, which derives from the general class "clothespin." We might (p. 44) formulate the view thus: A token artifact has the function of a certain performance just in case the artifact belongs to a type whose function is to perform in that way.

While I think it is right that function statements, whether in the natural or the artifactual realm, are primarily about types and only derivatively about tokens, ${ }^{17}$ this formulation of artifact function simply displaces to types of the very same worries discussed earlier. How does a type of artifact get its function? Saying that it is typical of, for instance, the kind "clothespin" that it fastens clothes to a line inherits all the problems of paper clips, fire extinguishers, and malfunctioning artifacts. My point is not that type membership is irrelevant but, rather, that appeal to it will not solve the serious difficulties that beset a conventionalist construal of artifact function.

\section{An Etiological Account of Function}

Intentionalism's strength is conventionalism's weakness, and vice versa. According to intentionalism, the maker's idea of the artifact's purpose sets the standard against which to measure its actual performances, thereby allowing one to identify and explain deformity, breakage, misuse, and idiosyncratic uses/performances. The problem is that intentionalism locates this standard in the maker's psychology without any plausible account of how the standard gets inscribed in or otherwise transferred to the object. While conventionalism gets function out of the maker's head and into the public realm of actual practices, it does so at the cost of normativity. As we have seen, conventionalism cannot distinguish between what an artifact is supposed to do and what it merely happens to do.

What we need is an account of artifact function that offers the benefits of each view without the attendant disadvantages. This is just what an etiological account of artifact function does. An etiological account of function focuses on the natural history of the thing in 
question (usually a trait), and in particular on how it got there ${ }^{18}$ Such an account is appealing in that it provides a normative conception of function, but it does so naturalistically-and, without appeal to its maker's intentions-by employing our best scientific theories, in particular, natural selection.

Here are the bare bones of an etiological account (noting that accounts of biological function tend to focus on traits of an organism). A trait's performance counts as the trait's function just in case this type of trait was selected for this kind of performance. Not just any performance counts as a trait's function: only that performance that enabled the ancestors (of the organism bearing the trait) to survive and reproduce is the function that is biologically "proper" to that trait. To say that a trait's performance is its proper function is to say that the trait has a history where it was selected for that performance. We might formulate the general idea thus: A trait has the (proper) function of a particular kind of performance in an organism just in case the trait has been selected for because that kind of trait has successfully performed in this way in the past. (Talk of "selection" in the biological realm, it should be noted, is shorthand for a process of elimination: (p. 45) organisms bearing the trait survive because the trait makes them especially suited to survive in the prevailing environment. To say that the trait in question was "selected for," on this account, simply means that the trait is [a] heritable and [b] contributed to the fitness of the organism's ancestors in virtue of performing in that way.)

What Ruth Millikan and philosophers following her call proper function is the performance that the trait in question ought to effect, even though the trait may not in fact live up to this; an item need not serve its function in order to have that function. ${ }^{19}$ It does not matter that a token heart, for instance, does not for whatever reason pump blood, because hearts were selected for this performance and this makes pumping blood their proper function. Selection history sets the norms that determine this purposive sense of function. It is because something has the right kind of history that it can be said to have a certain purpose. In Millikan's words, "items have functions when their being there depends on reproduction from ancestors having similar traits, these traits having been causally efficacious in helping to produce these items, and these traits having been selected at some point in this history for their capacity to make this kind of contribution."20

This kind of account of function may appear ill-suited to artifacts since they, at least at first blush, would seem to lack selection history. This impression, however, is mistaken. We shall see that artificial selection bridges the apparent gap between natural selection and the development of artifacts.

Let us call bio-artifact any living thing at least part of whose material configuration is due to intentional shaping by humans through artificial selection. Artificial selection-that is, the modification of a species by encouraging certain traits over others-happens through selective breeding, which works in roughly the following way. We begin with variation within a given population. From this population, breeders select desired traits and then isolate organisms bearing these traits so as to prevent random mating and hence prevent entrance into the population of new genetic material. Breeders then limit mating to those 
organisms that exhibit the desired characteristics, thereby weeding out the undesired traits while fixing the desired ones. Through this process, domesticated plants and animals change over time to become better adapted to human needs and desires.

An etiological account works nicely for bio-artifacts. Recall: A trait has the (proper) function of a particular kind of performance in an organism just in case the trait has been selected for because that kind of trait has successfully performed in this way in the past. As evolutionary biologists routinely point out, since "select" means to pick a thing out from a group, properly speaking there is no selection taking place in natural selection. Artificial selection, on the other hand, is aptly named because the animal or plant breeder chooses desired traits and selects the desired individuals from a group in order to serve as breeding stock for the next generation. There is no breeder making choices in natural selection; instead, as noted earlier, there is just a process of elimination where certain organisms do not survive long enough to reproduce because they cannot compete for scarce resources with other organisms bearing survival-favoring attributes. ${ }^{21}$ Natural selection is called "natural selection" because the struggle for survival in nature has effects that are similar to the actions of breeders in artificial selection. Of course, with natural selection the production of variation is random, whereas with artificial (p. 46) selection the production of variation is often the result of rational planning - although not always-but this does not make any decisive difference to the process. It just means that in the case of artificial selection the modifications have a greater likelihood of success, which speeds up the process of change. The difference between the two kinds of selection, then, is one of degree rather than of kind. ${ }^{22}$ We shall see shortly how this pertains to artifacts and material culture, but first we must consider how the etiological understanding of function works for bio-artifacts.

Let us take the example of the Basset Hound. One obvious question to ask about these dogs is why they have such large pendant ears, especially since their considerable length diminishes air circulation, which makes them prone to infection. What is the function of these unusually elongated flaps? On the etiological account, what we need to know is the selection history of the hound's ears: for what performance was this trait selected? The abbreviated answer is that the Basset Hound was bread for tracking small game, which explains its short legs and also its long ears that, along with its dewlap, trap and hold scent. So, whereas the human outer ear was selected to collect sound, the Basset's elongated flap was selected to collect smells. Though this may have been the ear flaps' original function, we have not used these dogs for hunting, at least not in the United States, for a very long time. What, then, explains the continued presence of this trait? ${ }^{23}$ There is still selection pressure on the breed to maintain the long ears-that is, breeders still isolate populations and limit mating to those hounds with the desired characteristics-but the motives for selection have changed. Nowadays Bassets are bread primarily for their mild temperament and cuteness, where long ears contribute significantly to the latter. This is to say that although the trait of long ears was initially selected for one purpose, namely trapping scent, it is now actively maintained for another purpose, namely cuteness or some variant of this aesthetic function. 
The important thing about the Bassett Hound is that while there has been no morphological modification of the trait (long floppy ears), the proper function of the trait has shifted. The long ears have been "exapted," to take a term from Stephen J. Gould and Elisabeth Vrba: they were selected for one performance but have been co-opted for another. ${ }^{24}$ But because there is selection pressure for this new role, it still counts as a proper function in Millikan's sense.

As historian George Basalla argued, artifact development occurs in a manner that is similar in important respects to artificial selection. ${ }^{25}$ Although it is common to portray progress in the artifactual realm-whether art or technology-as propagated by genius inventors or artists making great leaps forward, Basalla and others argue that this is not in fact how artifacts develop. ${ }^{26}$ Most quotidian artifacts-like wheels, hammers, forks, and pencils-have arisen slowly through the cumulative effects of small improvements-where adaption to human needs and desires is the standard against which improvement is measured. Even the seemingly most novel inventions and original artistic creations always centrally involve modification of some existing artifact. Indeed, inventors and artists work by perceiving inadequacies and failures in existing artifacts and then making modifications so that they will better suit our needs. ${ }^{27}$

The proposal, then, is that artifacts acquire functions in the same manner as bio-artifacts, namely through artificial selection. As with bio-artifacts, we begin with variation (p. 47) in the population of artifacts. Just consider the diversity of our artifactual world, some of which arises through invention, some through deliberate modification, and some by chance. From this manifold, desired traits are selected by inventors, users, and manufacturers. As with bio-artifacts, the criterion for selection is the artifact's "success," where this means not that the artifact performs as its maker intended but, rather, that its performances are deemed valuable by some members of the society. ${ }^{28}$ In this way "fitness" is determined by how well the artifact fulfills human needs and desires, where these may be medical, economic, political, aesthetic, and so forth. When a given artifact meets these needs and desires better than its competitors, it is selected over competitors for replication and distribution. As the artifact is used, its users may find it wanting improvement or their needs may change, and so modifications are introduced and passed on to subsequent generations. In this way, artifacts evolve, although it should always be remembered that what we call "progress" is relative to human needs and desires, which can also change radically over time; just as with biology, it is not "progress" in any absolute sense.

This evolutionary model accurately reflects the actual development of ordinary tools and utensils such as, for instance, the table fork. ${ }^{29}$ The table fork was not invented by a particular individual at a precise moment in human history but, rather, developed slowly out of modifications to other utensils, especially the knife. Some think that the fork was born out of frustrations with the knife's inadequacy in steadying food while carving and in lifting food from a boiling pot. The first forks had only two prongs that were widely spaced in order to facilitate carving and serving. From these serving forks developed forks with three and eventually four tines that are better suited to conveying certain kinds of food to the mouth. There was not some moment in history when the table fork was contrived ex 


\section{Artifacts and Their Functions}

nihilo by an ingenious inventor; rather, like so many artifacts, it developed slowly through the accretion of very small changes to already extant artifacts.

Art historian Heinrich Wölfflin famously wrote, "Not all things are possible at all times."30 Although the remark was restricted to one rarified kind of artifact-namely artworksWölfflin's point holds for any kind of artifact whatsoever, including forks (it also holds, noncoincidentally, for organisms). All artifacts are inextricably imbricated in a specific historical and cultural context which, borrowing another concept from biology, we might refer to as a niche: a specific set of environmental properties that are necessary-but not sufficient-for an artifact's survival (that is, for its being selected for reproduction). Just as organisms are remarkably adapted to animate and inanimate dimensions of their environments, so artifacts are adapted to specific properties of theirs: in particular, to available material and intellectual resources (including available technologies); to customs, practices, and social relations; and to existing material culture, that is, to other artifacts. However, we ought not to think of the interaction between artifact and environment as merely one-way; rather, artifacts also shape the very environments to which they adapt. ${ }^{31}$

The concept of niche, which is borrowed from ecology, can be understood in two importantly different senses. In the classical sense introduced by Charles Elton in 1927, a niche exists independently of its occupants and has specific requirements to which the (p. 48) occupants adapt. ${ }^{32}$ Biologist Richard Lewontin argues that the relationship between organism and its environment should be conceived differently; instead, as a dynamic and reciprocal relationship between organism and environment, where organisms and environment are co-constructive. ${ }^{33}$ According to this constructivist view of evolution, organisms shape their environment at the same time that the environment shapes them. This is understood as an ongoing process in which multiple species are in a constant process of shaping their environment in complex interactions that in turn produce changes in the species, where these changes introduce further complex and interactive changes in the environment, and so on. ${ }^{34}$ The upshot for an evolutionary account of function is that we should conceive of functions not as performances or activities that once-and-for-all meet pre-existent environmental demands but, rather, as a dynamic feedback loop where continually shifting performances shape the demands that these performances meet. ${ }^{35}$

This interactive picture of the relationship between artifact and environment provides an accurate and subtle understanding of the evolution of the human-made material world and so, I suggest, of artifact function. ${ }^{36}$ To begin with, a given artifact's development depends on both existing artifacts and practices, and also begets significant changes in other kinds of artifacts and in practices. Consider, again, the table fork. The fact that the fork speared food more efficiently than knives relieved the latter of the need for a pointed tip, which was also undesirable owing to its resemblance to weapons, hence the appearance of the blunt-tipped table knife in Europe. ${ }^{37}$ Further, people tend to prepare foods that are appropriate to the utensils that they have, and so cuisine adapts to artifacts for eating, as do table manners. ${ }^{38}$ The introduction of new foods may require changes in utensils, which can in turn motivate changes in table manners and also the development of new skills for using the utensils (and with new skills come changes in musculature and brain, as Lam- 
bros Malafouris shows in his compelling study). ${ }^{39} \mathrm{~A}$ table fork is not useful tout court but useful only in certain contexts at certain times, and only for certain foods, and only to those who have developed the skills to wield one. A table fork would not have been useful to, for instance, Homo habilis, nor is it useful to, for instance, most Indians, much to Oprah Winfrey's chagrin. ${ }^{40}$ The table fork continues to be reproduced in certain parts of the world-that is, is selected for replication-because the things it can do are valued by people in these areas, people whose cuisine and material culture call for a table fork and who have cultivated the skills required to manipulate it properly.

Like most artifacts, table forks have many capacities for which they are often used: whisking eggs, mashing soft fruit, crimping pie crusts before baking, lowering food into or fishing it from hot liquids, pulling pickles out of a jar, testing meats and vegetables for tenderness, back scratching, weeding, and so on (Disney's Ariel, a.k.a. the "Little Mermaid," famously uses a fork as a hair comb). None of these alternate uses, however, is a table fork's proper function. A fork has a proper function that was not bestowed upon it either by its maker (inventor, designer, or manufacturer) or by individual users. Rather, the fork's selection history, which should always be understood as niche relative, sets the norms that determine its proper function: viz., the activity of spearing food (p. 49) and conveying it to the mouth, an activity for which its ancestors bearing this capacity were selected and reproduced.

\section{Conclusion: Some Hard Cases}

The problem remains that talk about artifacts in teleological terms can easily appear to ascribe human characteristics to mere objects. This raises the troublesome question of justification. Can these useful and enlightening ways of speaking be acquitted of the charge of anthropomorphism? They can so long as they are understood aright; that is, where function is construed on the etiological and ecological model proposed in this chapter.

While one might accept this etiological account in the case of ordinary artifacts, one might nevertheless resist applying it to the kinds of artifacts that are thought to be sui generis; for example, inventions, artworks, and other things that seem to be, as Millikan puts it, "very new under the sun." To lump artworks and inventions in with forks, chairs, and other quotidian artifacts could seem to flagrantly disregard these artifacts' uniqueness and originality. Famous inventions afford a slightly neater case.

Consider the phonograph, as pure a case of invention as any. We credit Thomas Edison with its invention in 1877, although some twenty years earlier Leon Scott de Martinville of France had come up with a "phonoautograph" that recorded sounds but could not reproduce them. And a few months before Edison's patent another Frenchman-Charles Cros-had created a machine that both recorded and reproduced sounds, although sadly for his legacy he had no patent. These were all prototypes upon which Edison built. Although Edison did not intend his "invention" for this purpose, the function of the phonograph quickly became playing music for personal entertainment. Between 1900 and the 


\section{Artifacts and Their Functions}

present, a series of radical modifications were introduced and either reproduced or eventually eliminated, depending on how well they served the purpose of personal musical entertainment.

The phonograph then went through a variety of modifications that were better adapted to this purpose:

- It went from playing a cylinder in the 1910s to playing discs of varying shapes, sizes, and materials (hard rubber, shellac, and celluloid were all tried before it was determined that vinyl was best suited to our purposes).

- A variety of drive systems were tried for turntables (wheel drives, belt drives, and direct drives).

- Many pickup systems were tried: crystal, ceramic, and magnetic cartridges.

- A variety of arms were tried.

Modifications to the phonograph were introduced, and some of these were selected over others based on their fitness - that is, based on how well they fulfilled human needs (p. 50) and desires. These modifications were then reproduced-that is, introduced into the next generation of phonograph, and in this way the phonograph adapted to people's needs. However, keeping in mind Lewontin's point about constructivism, we should note that people's needs are constantly changing, in part in response to changes in the phonograph and related artifacts. With the introduction of digital media in the 1980s, and especially as these became better adapted for personal use (cheaper, less cumbersome, easier to use), the phonograph's function changed. Nowadays the biggest consumers of "phonographs" and vinyl records are audiophiles who prefer the analog format for aesthetic reasons, and disc jockeys who prefer this medium because it allows for direct manipulation: with a record one can move the stylus, accelerate or decelerate the turntable, or reverse its direction, thereby allowing for cueing, phrasing, beat juggling, scratching, needle drops, phase shifting, and other DJ techniques. So, as with the Basset Hound's ears and the table knife, we can say that the phonograph has been exapted for this new function; that is, this type of artifact is now actively maintained because of these new performances. The phonograph has a new function, one that its inventor never would have-to Wölfflin's point, never could have-dreamed of.

Every element of artificial selection does not extend to the artifactual realm. There are, of course, important differences that must be considered: for instance, artifacts are not capable of reproducing themselves; there is no genetic basis for inheritance; and there is no speciation in this realm. Any evolutionary of artifacts must acknowledge and accommodate these differences. However, thinking that the basic features of artificial selection apply to the artifactual realm, we do select artifacts that are best suited to certain tasks, reject those that are less well-suited, and modify surviving artifacts so that they perform their tasks better. Artifact types are modified and passed down over generations, and their replication is contingent upon their success in satisfying human needs, and those

Page 16 of 21

PRINTED FROM OXFORD HANDBOOKS ONLINE (www.oxfordhandbooks.com). (C) Oxford University Press, 2018. All Rights Reserved. Under the terms of the licence agreement, an individual user may print out a PDF of a single chapter of a title in Oxford Handbooks Online for personal use (for details see Privacy Policy and Legal Notice). 
needs in turn respond to, and in some cases are generated by, changes in the humanmade material world.

\section{Bibliography}

Alexander, Christopher. Notes on the Synthesis of Form. Cambridge, MA: Harvard University Press, 1964.

Basalla, George. The Evolution of Technology. Cambridge: Cambridge University Press, 1989.

(p. 53) Baxandall, Michael. Patterns of Intention: On the Historical Explanation of Pictures. Reprint edition. New Haven, CT: Yale University Press, 1987.

Coffin, Sarah D., Ellen Lupton, Darra Goldstein, and Barbara Bloemink. Feeding Desire: Design and the Tools of the Table, 1500-2005. New York: Assouline, 2006.

Dennett, Daniel C. "The Interpretation of Texts, People and Other Artifacts." Philosophy and Phenomenological Research 50 (1990): 177-194.

Gould, Stephen Jay, and Elisabeth S. Vrba. "Exaptation-a Missing Term in the Science of Form." Paleobiology, 1982, 4-15.

Hodder, Ian. Entangled: An Archaeology of the Relationships between Humans and Things. Malden, MA: Wiley-Blackwell, 2012.

Lewontin, Richard. "Sociobiology as an Adaptationist Program." Behavioral Science 24, no. 1 (1979): 5-14.

Malafouris, Lambros. How Things Shape the Mind: A Theory of Material Engagement. Cambridge, MA: MIT Press, 2013.

Millikan, Ruth Garrett. "Wings, Spoons, Pills, and Quills: A Pluralist Theory of Function.” Journal of Philosophy 96, no. 4 (1999): 191.

Neander, Karen. "The Teleological Notion of Function." Australasian Journal of Philosophy 69, no. 4 (1991): 454-468.

Petroski, Henry. The Evolution of Useful Things: How Everyday Artifacts-From Forks and Pins to Paper Clips and Zippers-Came to Be as They Are. Reprint edition. New York: Vintage, 1994.

Preston, Beth. "Why Is a Wing Like a Spoon? A Pluralist Theory of Function." Journal of Philosophy 95, no. 5 (1998): 215-254.

Pye, David. The Nature and Aesthetics of Design. London: A\&C Black, 2000.

Wilson, Bee. Consider the Fork: A History of How We Cook and Eat. New York: Basic Books, 2013. 


\section{Notes:}

(*) I would like to thank Ivan Gaskell for exchanging ideas about this topic long before he and Sarah Anne Carter invited me to contribute to this volume; and Allison Wylie, Walter Sinnott-Armstrong, Barbara Montero, and John Kulvicki for helpful comments on earlier drafts of this chapter. I presented partial drafts of this chapter at CUNY Graduate Center, Mt. Holyoke, and the Bard Graduate Center, and I am grateful for the many helpful comments I received there.

(1.) Daniel C. Dennett, “The Interpretation of Texts, People and Other Artifacts," Philosophy and Phenomenological Research 50 (October 1, 1990): 177-194.

(2.) Ian Hodder, Entangled: An Archaeology of the Relationships between Humans and Things, 1st ed. (Malden, MA: Wiley-Blackwell, 2012), 11.

(3.) Ibid.

(4.) G. E. M. Anscombe, Intention, 2nd ed. (Cambridge, MA: Harvard University Press, 2000).

(5.) Michael Baxandall, Patterns of Intention: On the Historical Explanation of Pictures, Reprint edition (New Haven, CT: Yale University Press, 1987), 25 ff.

(6.) Ruth Garrett Millikan, "Wings, Spoons, Pills, and Quills: A Pluralist Theory of Function," The Journal of Philosophy 96, no. 4 (April 1999): 192-193.

(7.) Valerie Hardcastle, "On the Normativity of Functions," in Functions: New Essays in the Philosophy of Psychology and Biology, ed. Mark Perlman (Oxford: Oxford University Press, 2002).

(8.) Paul E. Griffiths, "Functional Analysis and Proper Functions," in Function, Selection, and Design, ed. David J. Buller (Albany: SUNY Press, 1999).

(9.) David Pye, The Nature and Aesthetics of Design (London: A\&C Black, 2000), 12.

(10.) Hardcastle, “On the Normativity of Functions," 144.

(11.) Larry Wright, “Functions,” The Philosophical Review 82, no. 2 (April 1973): 139.

(12.) Henry Petroski, The Evolution of Useful Things: How Everyday Artifacts-From Forks and Pins to Paper Clips and Zippers-Came to Be as They Are, Reprint edition (New York: Vintage, 1994), 51.

(13.) Christopher Boorse, "A Rebuttal on Functions," in Functions: New Essays in the Philosophy of Psychology and Biology, ed. André Ariew, Robert Cummins, and Mark Perlman (Oxford: Oxford University Press, 2002), 63-112.

(14.) Thanks to Walter Sinott-Armstrong for making this point. 
(15.) Robert Cummins, "Functional Analysis," The Journal of Philosophy 72, no. 20 (November 20, 1975): 741.

(16.) Wright, "Functions."

(17.) Karen Neander, “The Teleological Notion of 'Function,'” Australasian Journal of Philosophy 69, no. 4 (1991): 454-468.

(18.) The idea that one must know a thing's history in order to properly ascribe a function to it begins with Wright, "Functions," although etiology is only half of Wright's conception of function. The etiological account is more fully developed by Ruth Millikan: Ruth Garrett Millikan, Language, Thought, and Other Biological Categories: New Foundations for Realism (Cambridge, MA: MIT Press, 1987); Ruth Garrett Millikan, White Queen Psychology and Other Essays for Alice (Cambridge, MA: MIT Press, 1993); Millikan, "Wings, Spoons, Pills, and Quills." For helpful review and analysis of the terrain, see Beth Preston, "Why Is a Wing Like a Spoon? A Pluralist Theory of Function," Journal of Philosophy 95, no. 5 (1998): 215-254.

(19.) Millikan, Language, Thought, and Other Biological Categories, 29.

(20.) Millikan, White Queen Psychology and Other Essays for Alice, 41.

(21.) Ernst Mayr, What Evolution Is, Reprint edition (New York: Basic Books, 2002), $115 \mathrm{ff}$.

(22.) Preston, "Why Is a Wing Like a Spoon?”; Daniel C. Dennett, Darwin's Dangerous Idea: Evolution and the Meanings of Life, Reprint edition (New York: Simon \& Schuster, 1996), 317-318.

(23.) See Millikan, White Queen Psychology and Other Essays for Alice, 47ff. on the distinction between being selected for and being actively maintained for.

(24.) Stephen Jay Gould, “Exaptation: A Crucial Tool for an Evolutionary Psychology,” Journal of Social Issues 47, no. 3 (1991): 43-65; Stephen Jay Gould and Elisabeth S. Vrba, "Exaptation-a Missing Term in the Science of Form," Paleobiology, 1982, 4-15.

(25.) George Basalla, The Evolution of Technology (Cambridge: Cambridge University Press, 1989). While there are proponents of an etiological account of artifact functionfor example, Preston, "Why Is a Wing Like a Spoon?" and to some extent Elder-most prefer to use natural selection as their model for how artifacts acquire proper functions. In the end it does not matter much which model one chooses since the processes are analogous in the relevant respects. Artifact evolution is simply closer to artificial selection in the sense that it is humans, rather than nature, doing the selecting (where, as noted earlier, the claim that nature "selects" should be understood as a process of elimination).

(26.) Basalla, The Evolution of Technology; Petroski, The Evolution of Useful Things. 
(27.) On the central role of failure in invention and the evolution of artifacts, see Pye, The Nature and Aesthetics of Design; Petroski, The Evolution of Useful Things, chap. 2;

Christopher Alexander, Notes on the Synthesis of Form (Cambridge, MA: Harvard University Press, 1964).

(28.) Basalla, The Evolution of Technology, chap. 1 and 2.

(29.) Sarah D. Coffin et al., Feeding Desire: Design and the Tools of the Table, 1500-2005 (New York: Assouline, 2006); Petroski, The Evolution of Useful Things; Bee Wilson, Consider the Fork: A History of How We Cook and Eat (New York: Basic Books, 2013).

(30.) Heinrich Wölfflin, Principles of Art History: The Problem of the Development of Style in Later Art (New York: Dover, 1950).

(31.) Thanks to Lambros Malafouris and John Robb for pressing me to develop this point.

(32.) Charles S. Elton, Animal Ecology (Chicago: University of Chicago Press, 2001); Kim Sterelny, Sex and Death: An Introduction to Philosophy of Biology (Chicago: University of Chicago Press, 1999), chap. 11.

(33.) Richard Lewontin, "Sociobiology as an Adaptationist Program," Behavioral Science 24, no. 1 (January 1, 1979): 5-14; Richard Lewontin, The Triple Helix: Gene, Organism, and Environment (Cambridge, MA: Harvard University Press, 2002), chap. 2.

(34.) Lewontin, The Triple Helix, chap. 2.

(35.) My understanding here has been greatly influenced by Kim Sterelny and Paul E. Griffiths, Sex and Death: An Introduction to Philosophy of Biology, 1st ed. (Chicago: University of Chicago Press, 1999), chap. 11.

(36.) Hodder, Entangled; Lambros Malafouris, How Things Shape the Mind: A Theory of Material Engagement (Cambridge, MA: MIT Press, 2013).

(37.) Petroski, The Evolution of Useful Things, chap. 1.

(38.) As Bee Wilson discusses, the invention of pottery worked in tandem with the science of agriculture to change diets-allowing for the preparation of mushy grains-and also cooking methods, since pottery allowed for boiling. Wilson, Consider the Fork, chap. 1.

(39.) Malafouris, How Things Shape the Mind.

$\left({ }^{40 .}\right)$ At the start of a meal on her recent visit to India, Oprah asked, "I heard that some Indian people eat with their hands still." It was the "still" that, I think, gave many offense because it was taken, to my mind reasonably, to imply that Western cutlery is more advanced in some absolute sense. But table forks are not an advance over eating with hands or chopsticks; table forks simply are not useful to most Indians, in part because Indian cuisine has developed in a way that does not call for forks, and also because most Indians 


\section{Artifacts and Their Functions}

have not cultivated the skills required to manipulate table forks properly. Inserted into a foreign context (i.e., a context that is not its niche), a table fork is quite useless.

\section{A. W. Eaton}

A. W. Eaton is Associate Professor of Philosophy at the University of Illinois at Chicago. 